\title{
Bi-rotary Maps of Negative Prime Characteristic
}

\author{
Antonio Breda d'Azevedo, Domenico A. Catalano and Jozef Širáň
}

\begin{abstract}
Bi-orientable maps (also called pseudo-orientable maps) were introduced by Wilson in the 1970s to describe non-orientable maps with the property that opposite orientations can consistently be assigned to adjacent vertices. In contrast to orientability, which is both a combinatorial and topological property, bi-orientability is only a combinatorial property. In this paper we classify the bi-orientable maps whose localorientation-preserving automorphism groups act regularly on arcs, called here bi-rotary maps, of negative prime Euler characteristic. Unlike other classification results for highly symmetric maps on such surfaces, we do not use the Gorenstein-Walter result on the structure of groups with dihedral Sylow 2-subgroups.
\end{abstract}

Mathematics Subject Classification. 05C10, 05C15, $05 \mathrm{C} 25$.

Keywords. Prime characteristic, Pseudo-orientable maps.

\section{Introduction}

A map is a cellular embedding of a connected graph on a compact surface. If the map is orientable, meaning that its carrier surface is orientable, then a choice of its global orientation induces a local orientation around each vertex. This set of local orientations has the obvious property that when moving along an arbitrary edge within a sufficiently narrow band on the carrier surface of the map, the local orientations at the two vertices incident to the edge agree.

In this paper, we will consider maps exhibiting the opposite phenomenon, apparently first considered in Wilson [21]. Call a map $M$ on a (not necessarily orientable) surface bi-orientable if it admits an assignment of local orientations around vertices on the surface in such a way that when traversing an edge between a pair of incident vertices as above, the local orientations at the two vertices disagree. We note that such maps were called pseudo-orientable in the original paper [21].

Orientable and bi-orientable maps are related by means of Petrie duality. Namely, a map is bi-orientable if and only if its Petrie dual is orientable, and, 
for an orientable map, its Petrie dual is orientable if and only if its underlying graph is bipartite. (These two facts are likely to have folklore character but we nevertheless include short proofs in Sect. 2.) This could leave an impression that bi-orientable maps do not need any further attention since their study can be reduced to the investigation of orientable maps. Such a judgement, however, is inadequate when considering maps on a given surface, as Petrie duality need not preserve carrier surfaces.

We will be interested in bi-orientable regular maps on a given surface which, in addition, are 'highly symmetric'. Deferring a detailed explanation to Sect. 2, an automorphism of a map may be identified (up to degenerate cases) with a permutation of the incident vertex-edge pairs that extends to a self-homeomorphism of the carrier surface, preserving incidence between vertices, edges and faces of the map. It turns out that the group of all those automorphisms of a (bi-)orientable map which preserve the local orientations acts semi-regularly on the set of incident vertex-edge pairs of the map. If this action is transitive, and hence regular, the corresponding orientable map is known as orientably regular or rotary; in the bi-orientable case such a map will be called bi-rotary.

Orientably regular and bi-rotary maps intuitively exhibit the highest 'level of symmetry' with respect to preservation of local orientations around vertices. An even higher 'level of symmetry', however, arises when the automorphism group of a map acts transitively - and hence regularly — on the flags (i.e. mutually incident vertex-edge-side triples), and such maps will be called fully regular. We note here that terminology regarding maps with high 'level of symmetry' has not been unified (see also Širán̆ [20]) and we will be freely using the term regular map to refer to any of the three kinds of maps introduced in this and the previous paragraphs if no confusion is likely.

As we shall see in Sect. 2, the theory of regular maps is closely related to presentations of groups generated by specific pairs or triples of generators, triangle groups, Riemann surfaces, and even to Galois theory. We refer to Jones and Singerman [17] for an overview of these and other connections that have motivated a considerable amount of work in classification of regular maps, either on a particular surface, or with a given underlying graph, or else having a prescribed automorphism group. Here we will concentrate on regular maps on a given carrier surface, and we will very briefly summarize the corresponding knowledge, referring to the survey [20] for results in the other two directions.

Leaving the well-known spherical, projective-planar and toroidal cases aside (cf. e.g. [12,20]), Euler's formula implies that every surface of negative Euler characteristic carries only a finite number of regular maps. Complete lists of orientably and fully regular maps on surfaces of negative Euler characteristic $\chi \geq-12$ were compiled as a result of a collective effort of a number of authors and we refer to [20] for historical details. The first computer-aided classification of such maps on surfaces with $\chi \geq-30$ appeared in [7].

Breakthroughs started about a decade ago [4] with a classification of fully regular maps on non-orientable surfaces of Euler characteristic $\chi=-p$ for every prime $p$, followed by a result of a similar nature for $\chi=-p^{2}$ in 
[10] and $\chi=-3 p$ in [9], and by a classification of orientably and fully regular maps on orientable surfaces with $\chi=-2 p$ in $[1,11]$. A wealth of information on the structure of automorphism groups of orientably regular maps on a given surface was obtained in [11]. We also note that to the date of submission of this article a computer-aided classification of orientably and fully regular maps has been available for all surfaces of Euler characteristic $\chi \geq-600$, see Conder $[6]$.

To the best of our knowledge, apart from a census of pseudo-orientable regular maps and hypermaps of Euler characteristic at least -16 given in [3], no classification results as above have been known for bi-rotary maps, despite being a natural counterpart to orientably regular maps. The relationship between the two kinds of maps, however, is much deeper. Indeed, as we shall see in Sect. 2, they both arise as quotients of certain index-two subgroups of extended triangle groups.

In this paper, we derive a classification of bi-rotary maps on non-orientable surfaces of Euler characteristic $-p$ for an arbitrary prime $p$. A precise statement of this result, with presentations of the corresponding automorphism groups, is given in Theorem 6.1 in Sect. 6. Quite remarkably, in the proof of our classification results we do not rely on the deep result of Gorenstein and Walter [14] about the structure of finite groups with dihedral Sylow 2subgroups, which was used in the aforementioned results for regular maps on surfaces of Euler characteristic $-p,-p^{2},-2 p$ and $-3 p$. Instead, in the analysis of our groups it turned out to be sufficient to use the result of Huppert [15] on the solvability of products of groups close to cyclic groups combined with the Fitting subgroup approach of Conder [10].

The paper is organized as follows. In Sect. 2, we sum up basic concepts and results concerning regular, rotary, bi-orientable and bi-rotary maps; a corresponding algebraic setting is developed in Sect. 3. Preliminary observations of algebraic nature about bi-rotary maps of Euler characteristic $-p$ for an odd prime $p$ in the case when $p$ divides the order of the automorphism group of the map are summed up in Sect. 4, followed by Sect. 5 containing a proof of the core part of our classification. The final Sect. 6 summarizes our results and includes further observations and remarks.

\section{Maps and Automorphisms}

In this section, we formalize the concepts and results mentioned in the Introduction and present a more detailed background on regular maps. The terminology and notation built in the course of our explanations will eventually lead to formulation of our main results.

As already stated, throughout by a map we mean a cellular embedding of a connected graph on some surface (a connected two-dimensional Hausdorff space in which every point has a neighbourhood homeomorphic to an open disc). For the most part, our surfaces will be compact and not necessarily orientable. A map will be called orientable (non-orientable) according to the orientability type of its supporting surface. 
Let $M$ be a map. Removing a sufficiently small open neighbourhood of one point from the interior of every face of $M$, one obtains a 2-complex $B(M)$ carrying the same underlying graph. Topologically, the 2-complex $B(M)$ can be visualized by imagining that vertices of the embedded graph are contained in the interiors of sufficiently small pairwise disjoint closed discs and edges are contained in the interiors of sufficiently narrow closed bands attached to the discs. We will say that $B(M)$ is the band complex of $M$.

We continue by constructing a new 2-complex $K$ with boundary by introducing a half-turn twist to every closed band of $B(M)$ surrounding an edge, as illustrated in Fig. 1. Identifying boundary components of $K$ with boundaries of pairwise disjoint closed discs one obtains a new map $M_{\mathrm{tw}}$ on some surface, with the same underlying graph, so that $K=B\left(M_{\mathrm{tw}}\right)$ can be identified with a band complex of $M_{\mathrm{tw}}$. The new map $M_{\mathrm{tw}}$ is known as the Petrie dual of $M$ and is standardly denoted by $P(M)$. Combinatorially, face boundaries of the Petrie dual are obtained by 'left-right' or 'zigzag' walks on the original map. Note that the Petrie operator $M \mapsto P(M)$ on maps is involutory, since the above description of $P(M)$ allows one to identify the map $P(P(M))$ with $M$ in an obvious way.

Let now $M$ be an oriented map, that is, a map on an orientable surface with a specified global orientation. The chosen orientation of the surface induces a local orientation around each vertex of $M$ and $B(M)$, so that when walking along an edge on a band the local orientations at the two vertices incident to the edge agree. Assume further that one keeps the local orientations in the small discs at vertices of $M$ and $B(M)$ in the above process of constructing $B\left(M_{\mathrm{tw}}\right)$ and $M_{\mathrm{tw}}$ by twisting bands. The map $M_{\mathrm{tw}}=P(M)$ has then the interesting property that when walking along an arbitrary edge on the corresponding (twisted) band, the local orientations at the two vertices incident to the edge disagree.

As already mentioned, maps with this property have been considered before in [21]. Diverting slightly from the terminology introduced therein, in general, a map $M$ on some surface (orientable or not) will be called bi-orientable

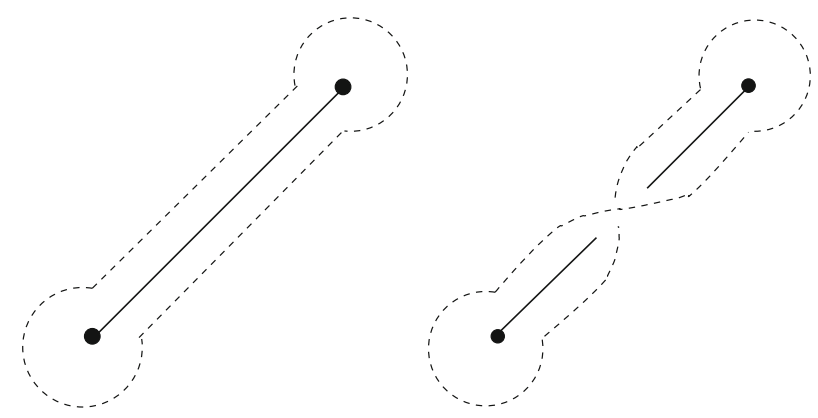

FigURE 1. Illustration of a half-turn twist of a band centred at an edge 
if it admits an assignment of local orientations on the surface around vertices of the underlying graph on the supporting surface in such a way that when walking along an edge on a sufficiently narrow surrounding band the local orientations at the two vertices incident to the edge disagree. A choice of local assignments as described above is a bi-orientation, making such a map bi-oriented.

Let $M$ be a (bi-)orientable map, by which we mean that $M$ is either orientable or bi-orientable. It is then easy to see that the Petrie dual $P(M)$ of $M$ is also (bi-)orientable. In somewhat more detail, we have:

Lemma 2.1. If $M$ is both orientable and bi-orientable, then so is $P(M)$. Moreover, $M$ is orientable but not bi-orientable if and only if $P(M)$ is bi-orientable and not orientable.

Proof. If $M$ is bi-orientable, then in $P(M)$ the local orientations inherited from $M$ agree when traversing an arbitrary edge along a narrow surrounding band. This means that the supporting surface of $P(M)$ admits a global orientation and hence is orientable. Conversely, if $P(M)$ is orientable then $M=P(P(M))$ is bi-orientable, as explained above. Our lemma is an obvious consequence of the two observations.

For completeness we include another result on Petrie duals that appears to be folklore.

Lemma 2.2. If $M$ is an orientable map, then $P(M)$ is orientable if and only if the underlying graph of $M$ is bipartite.

Proof. Assume that $P(M)$ is orientable. The maps $M$ and $P(M)$ share the same underlying graph. If this graph contained an odd cycle $C$, then orientability of $P(M)$ and the band-twisting construction of $P(P(M))=M$ imply that there would be a Möbius band surrounding $C$ in the supporting surface of $M$, contrary to the orientability assumption on $M$. This proves that orientability of $P(M)$ implies that its underlying graph is bipartite.

Conversely, if the underlying graph of an orientable map $M$ is bipartite, its vertices can be properly two-coloured, say, black and white. Such a colouring allows us to introduce, on the orientable carrier surface of $M$, clockwise local rotations on white vertices and counterclockwise local rotations on black vertices, making $M$ a bi-oriented map. Orientability of $P(M)$ then follows from Lemma 2.1.

We will now discuss automorphisms. Let $D$ be the set of arcs, that is, edges with a specified direction, of the underlying graph $\Gamma$ of a (bi-)orientable map $M$ on a carrier surface $\mathcal{S}$. For the purpose of this article one may identify arcs with incident vertex-edge pairs mentioned earlier. If $M$ is orientable, an orientation-preserving automorphism of $M$ is a permutation of $D$ forming an automorphism of $\Gamma$ that extends to an orientation-preserving self-homeomorphism of $\mathcal{S}$, and we let $\operatorname{Aut}^{+}(M)$ denote the group of all such orientationpreserving automorphisms of $M$. If $M$ is bi-orientable, a bi-orientation-preserving automorphism of $M$ is an automorphism of $\Gamma$ (represented as a permutation of $D$ ) that extends to a self-homeomorphism of $\mathcal{S}$ preserving all the local 
orientations at vertices in a chosen bi-orientation of $M$, and the corresponding group will be denoted $\mathrm{Aut}^{b}(M)$.

It is obvious that the stabilizer of any arc of a (bi-)orientable map $M$ under the action of $\mathrm{Aut}^{+}(M)$ or $\mathrm{Aut}^{b}(M)$ is trivial, giving semi-regular permutation groups on the arc set $D$ of $M$. In the case when the group $\operatorname{Aut}^{+}(M)$ happens to be a regular permutation group on $D$ we say that the map $M$ is orientably regular or rotary. Similarly, if $\mathrm{Aut}^{b}(M)$ is regular on $D$, we call the map $M$ bi-rotary. Loosely speaking, rotary and bi-rotary maps exhibit the 'highest level of symmetry while preserving orientation or bi-orientation'. In particular, such a map has all vertices of the same valency, say, $k$, and all faces are bounded by closed walks of the same length, say $m$; the map is then said to be of type $(k, m)$.

Note that $M$ can be rotary and bi-rotary at the same time and in such a case the groups $\mathrm{Aut}^{+}(M)$ and $\mathrm{Aut}^{b}(M)$ coincide only in the case when both are trivial; in all other cases the two groups are distinct, though they might be isomorphic. Another obvious consequence of the above definitions in connection with Petrie duality is:

Lemma 2.3. If $M$ is an orientable map, then $\operatorname{Aut}^{+}(M)=\operatorname{Aut}^{b}(P(M))$, and if $M$ is bi-orientable, then $\operatorname{Aut}^{b}(M)=\operatorname{Aut}^{+}(P(M))$.

A rotary or a bi-rotary map may also admit a symmetry that reverses local orientations. Assuming that the degree of our map is $k \geq 3$ to avoid trivialities, an orientation-reversing automorphism of a map may be identified with a permutation of its arc set that preserves incidence between vertices, edges and faces of the map and reverses (bi-)orientation at vertices. Rotary or bi-rotary maps admitting an orientation-reversing automorphism will be called reflexible. For such a map $M$ the group of all its automorphisms will be denoted $\operatorname{Aut}(M)$, and it is clear that it contains either $\mathrm{Aut}^{+}(M){\text { or } \mathrm{Aut}^{b}(M)}$ as a subgroup of index two; maps that are not reflexible will be called chiral.

The concepts of reflexibility and chirality have become standard in the theory of highly symmetric maps, although some of the corresponding terminology has not been unified; see e.g. [20]. To avoid misunderstandings, a map whose automorphism group acts regularly on flags, that is, on mutually incident vertex-edge-side triples, will be called fully regular, as in [8]. If not specified, the term 'regular map' may refer to any of rotary, bi-rotary, or fully regular maps, but we will only use it if no confusion is likely. Note that reflexible rotary and bi-rotary maps are both fully regular; the regular embedding of the Petersen graph in a projective plane shows that the reverse inclusion fails to hold.

\section{Regular and Bi-rotary Maps-Algebraically}

By a general theory of maps on surfaces as developed in [5], every map with least common multiple of vertex valencies and face boundary lengths equal to $k$ and $m$ can be obtained as a quotient of a tessellation $U(k, m)$ of a simply connected surface by $m$-gons, $k$ of which meet at every vertex, by a subgroup 


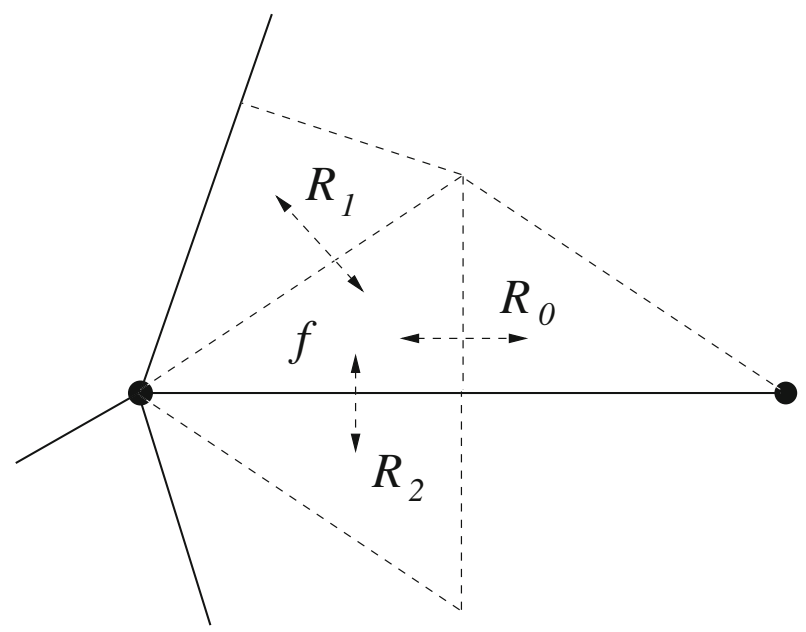

FiguRE 2. Action of the generating involutory automorphisms $R_{i}$ on a distinguished flag $f$

of the automorphism group of $U(k, m)$. This automorphism group is known to be the full $(2, k, m)$-triangle group $\Delta(k, m)$ with presentation

$$
\Delta(k, m)=\left\langle R_{0}, R_{1}, R_{2} \mid R_{0}^{2}, R_{1}^{2}, R_{2}^{2},\left(R_{0} R_{2}\right)^{2},\left(R_{1} R_{2}\right)^{k},\left(R_{0} R_{1}\right)^{m}\right\rangle .
$$

For $i \in\{0,1,2\}$ the generator automorphisms $R_{i}$ are to be interpreted as reflections in the three sides of some distinguished flag $f$, fixing its $j$-dimensional components for $j \neq i$ as indicated in Fig. 2. Invoking [5] again, a fully regular map of type $(k, m)$ then corresponds to a quotient $U(k, m) / N$ by a normal torsion-free subgroup $N$ and its automorphism group has a partial presentation

$$
\left\langle r_{0}, r_{1}, r_{2} \mid r_{0}^{2}, r_{1}^{2}, r_{2}^{2},\left(r_{0} r_{2}\right)^{2},\left(r_{1} r_{2}\right)^{k},\left(r_{0} r_{1}\right)^{m}, \ldots\right\rangle .
$$

Here and in all forthcoming presentations of groups we always assume that powers are true orders of the corresponding elements.

Conversely, given a presentation (3.2) of a group $G$ in terms of three involutions $r_{0}, r_{1}, r_{2}$, the corresponding fully regular map can be reconstructed by letting the (say, left) cosets of the subgroups $\left\langle r_{0}, r_{2}\right\rangle,\left\langle r_{1}, r_{2}\right\rangle$ and $\left\langle r_{0}, r_{1}\right\rangle$ be edges, vertices and faces, respectively; incidence between these building blocks is given by non-empty intersection. The map $M$ resulting this way will be denoted $\operatorname{Map}\left(G ; r_{0}, r_{1}, r_{2}\right)$, and the group $G$ acts as the automorphism group $\operatorname{Aut}(M)$ of this map simply by left multiplication. Without going into details, it follows from Bryant and Singerman [5] (cf. also Širán̆ [20]) that two such fully regular maps $\operatorname{Map}\left(G ; r_{0}, r_{1}, r_{2}\right)$ and $\operatorname{Map}\left(H ; s_{0}, s_{1}, s_{2}\right)$ are isomorphic if and only if there is a group isomorphism from $G$ onto $H$ taking $r_{i}$ onto $s_{i}$ for $i \in\{0,1,2\}$.

The group $\Delta(k, m)$ can have up to 7 subgroups of index two, depending on the parity of $k$ and $m$. To see this it is sufficient to realise that at least 
one of the generators $R_{i}, i \in\{0,1,2\}$, must lie outside of such a subgroup, and there are seven possibilities for this to happen. The situation when this is the case for all three generators is particularly important since then, letting $R=R_{0} R_{1}$ and $S=R_{1} R_{2}$, the subgroup is the (ordinary) $(2, k, m)$-triangle group $\Delta^{+}(k, m)$ with presentation

$$
\Delta^{+}(k, m)=\left\langle R, S \mid(R S)^{2}, S^{k}, R^{m}\right\rangle .
$$

Ordinary triangle groups are 'universal' for orientable regularity since by the classical results of Jones and Singerman [16] every orientably regular map of type $(k, m)$ can be identified with the quotient space $U(k, m) / N$, and, equivalently, with the quotient group $\Delta^{+}(k, m) / N$, for a suitable normal and torsionfree subgroup $N$ of $\Delta^{+}(k, m)$.

It turns out (cf. $[2,3])$ that bi-rotary maps also arise from index-two subgroups of full triangle groups. But let us have a more detailed look at bi-rotary maps first. Let $e$ be an arc of a bi-rotary map $M$ pointing into a vertex $u$ and let $G=\operatorname{Aut}^{b}(M)$. Coherence of $M$ implies that there exists an automorphism $z \in G$ such that $z(e)$ is the next arc at $u$ in the chosen local orientation around $u$. By the same token there is an automorphism $a \in G$ sending the arc $z^{-1}(e)$ onto its reverse arc; see Fig. 3. One may visualize the local action of $a$ and $z$, respectively, as a reflection in the axis of symmetry of the edge formed by the $\operatorname{arcs} z^{-1}(e)$ and $a z^{-1}(e)$, and a counterclockwise rotation about $u$ by one notch, taking the arc $z^{-1}(e)$ onto $e$. The situation, depicted in Fig. 3, also shows that the commutator $[a, z]=a z a z^{-1}$ (in the notation of [19]) acts locally as a counterclockwise rotation of the face $F$ incident with the arcs $e$ and $z^{-1}(e)$ by two notches, taking the arc $e$ onto the arc $[a, z](e)$. Note that there is no automorphism in $G=\operatorname{Aut}^{b}(M)$ taking $e$ onto $a z^{-1}(e)$ and preserving the face $F$ since such a mapping would have to reverse local orientations; hence the face length of $M$ must be even.

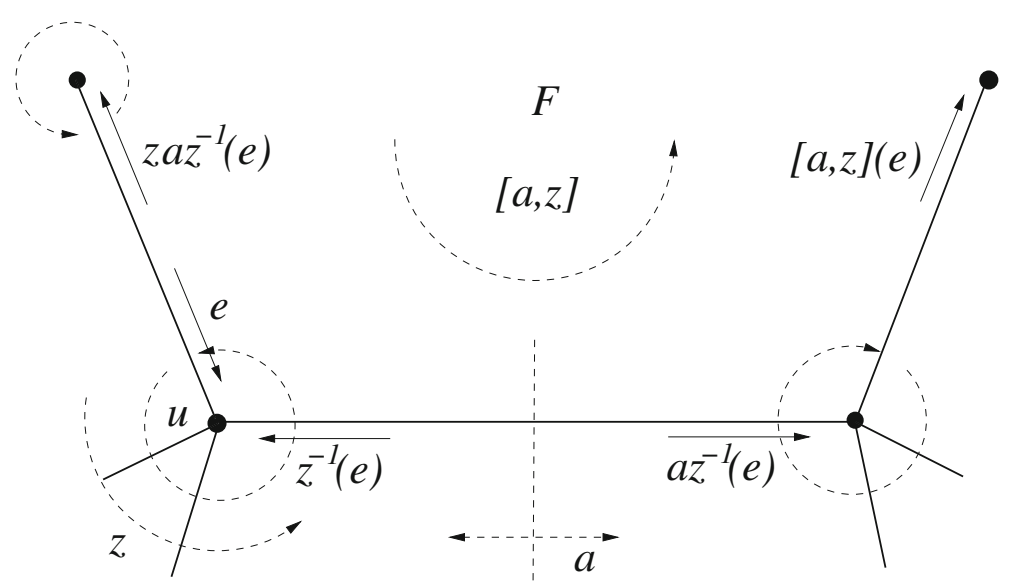

FIGURE 3. Local actions of the automorphisms $a, z$ and $[a, z]$ on a bi-rotary map 
Connectedness of the underlying graph of $M$ implies that $G$ is generated by $a$ and $z$. Moreover, if $M$ is of type $(k, m)$, then, as we saw, $m$ must be even and the group admits a partial presentation of the form

$$
G=\left\langle a, z \mid a^{2}, z^{k},[a, z]^{m / 2}, \ldots\right\rangle .
$$

Returning to quotients of full triangle groups, let $\Delta^{b}(k, m)$ be the subgroup of $\Delta(k, m)$ as presented in (3.1), generated by the elements $A=R_{0}$ and $Z=R_{1} R_{2}$. If $m$ is even, then $\Delta^{b}(k, m)$ is a subgroup of $\Delta(2, k, m)$ of index two and has a full presentation

$$
\Delta^{b}(k, m)=\left\langle A, Z \mid A^{2}, Z^{k},[A, Z]^{m / 2}\right\rangle .
$$

By the theory outlined in $[2,3]$, every bi-rotary map of type $(k, m)$ can be identified with the quotient map $U(k, m) / N$, and also with the quotient group $\Delta^{b}(k, m) / N$, for a suitable normal torsion-free subgroup $N$ of $\Delta^{b}(k, m)$. Note that if $M$ is a bi-rotary map with the group $G=$ Aut $^{b}(M)$ presented as in (3.4), we have an obvious epimorphism $\Delta^{b}(k, m) \rightarrow G$ taking $A$ and $Z$ onto $a$ and $z$, respectively.

The line of thought may here be reversed as in the case of fully regular maps. Namely, given a two-generator presentation of a group $G$ as in (3.4), the corresponding bi-rotary map $M$ may be obtained by taking elements of the group $G$ as arcs (left) cosets of the subgroups $\langle a\rangle$ and $\langle z\rangle$ as edges and vertices. Since the rotation induced by the commutator $[a, z]$ has two orbits on the boundary of the corresponding face, this time faces of $M$ are formed by pairs of cosets $g\langle[a, z]\rangle \cup g a\langle[a, z]\rangle$ for $g \in G$, with incidence defined by non-empty intersection again. For the map arising this way we will use the notation $\operatorname{Map}(G ; a, z)$ throughout; no confusion with the earlier notation for fully regular maps will be likely. A pair of bi-rotary maps $\operatorname{Map}\left(G_{1} ; a_{1}, z_{1}\right)$ and $\operatorname{Map}\left(G_{2} ; a_{2}, z_{2}\right)$ are isomorphic if and only if there is a group isomorphism $G_{1} \rightarrow G_{2}$ taking $a_{1}$ onto $a_{2}$ and $z_{1}$ onto $z_{2}$. Orientability of a map $\operatorname{Map}(G ; a, z)$ can also be decided in a simple way, observing that $\langle[a, z], z\rangle$ is a subgroup of $G$ of index at most 2 :

Lemma 3.1. A bi-rotary map $\operatorname{Map}(G ; a, z)$ is non-orientable if and only if $G=$ $\langle[a, z], z\rangle$.

Proof. If a bi-rotary map $M=\operatorname{Map}(G ; a, z)$ is orientable, then $\langle[a, z], z\rangle$ is a subgroup of $G$ of index 2 since both $[a, z]$ and $z$ are orientation preserving automorphisms of $M$ while $a$ is orientation reversing. Conversely, if $H=\langle[a, z], z\rangle$ is a subgroup of $G$ of index 2 , then $H$ is the subgroup of $G$ consisting of words in $a, z$ containing an even number of $a$ 's. Our earlier considerations then imply that the underlying graph of $M$ is bipartite, with cosets of the form $h\langle z\rangle$ and $h a\langle z\rangle=h\langle[a, z] \cdot z\rangle a$ representing vertices in the two parts (recall our assumption about true orders, in particular, of $a$ ). By Lemma 2.1 we know that the map $M^{\prime}=P(M)$ is orientable, and by Lemma 2.2 the map $P\left(M^{\prime}\right)=M$ is orientable.

From now on, all groups considered will be assumed to be finite. A (finite) bi-rotary map $M=\operatorname{Map}(G ; a, z)$ of type $(k, m)$, with $G$ presented as in (3.4), 
TABLE 1. Hyperbolic pairs $(k, m)$ with even $m$ and even $\nu=$ $\nu(k, m)$

\begin{tabular}{rrrrrrrrr}
\hline$k$ & $m$ & $\nu$ & $k$ & $m$ & $\nu$ & $k$ & $m$ & $\nu$ \\
\hline 3 & 8 & 24 & 4 & 8 & 8 & 6 & 6 & 6 \\
3 & 12 & 12 & 4 & 12 & 6 & 6 & 12 & 4 \\
3 & 24 & 8 & 5 & 4 & 20 & 8 & 8 & 4 \\
4 & 6 & 12 & 5 & 20 & 4 & & & \\
\hline
\end{tabular}

has $|G| / k$ vertices, $|G| / 2$ edges and $|G| / m$ faces. The Euler characteristic $\chi=$ $\chi(M)$ of $M$, defined as the Euler characteristic of the carrier surface of $M$, is then given by $\chi=|G|\left(\frac{1}{k}-\frac{1}{2}+\frac{1}{m}\right)$. If $\chi<0$, that is, if the type $(k, m)$ is hyperbolic, meaning that $\frac{1}{k}+\frac{1}{m}<\frac{1}{2}$, Euler's equation can be rewritten in the form

$$
|G|=\nu(k, m)(-\chi) \quad \text { where } \quad \nu(k, m)=\left(\frac{1}{2}-\frac{1}{k}-\frac{1}{m}\right)^{-1}=\frac{2 k m}{k m-2 k-2 m} .
$$

Recall that our aim is to classify all bi-rotary maps $M$ of type $(k, m)$ with Euler characteristic $\chi(M)=-p$ for $p$ a prime. In the light of the theory outlined in this section our task can be formally stated as follows:

Classify all the two-generator presentations of groups $(G ; a, z)$ of the form (3.4) and satisfying $|G|=\nu(k, m) p$, up to equivalence of generating pairs $(a, z)$ given by conjugacy within the automorphism group $\operatorname{Aut}(G)$ of $G$.

This will be done in the forthcoming sections together with a discussion of related algebraic issues. Note that for odd $p$ the above bi-rotary maps are automatically non-orientable.

\section{Preliminary Observations}

Throughout, let $M=\operatorname{Map}(G ; a, z)$ be a bi-rotary map of a hyperbolic type $(k, m)$ for even $m$, with a given Euler characteristic $\chi<0$. Notice that negative characteristic implies $m \geq 4$ and so the order $m / 2$ of $[a, z]$ is at least 2 , implying that $G$ is non-Abelian. Euler's equation (3.6) suggests that the case when $\nu(k, m)$ is an integer deserves special consideration, which we will do next.

It is well known that the largest value of $\nu(k, m)$, taken over all hyperbolic pairs $(k, m)$ with $m$ even, is 24 , attained for the unique pair $(k, m)=(3,8)$. It is also easy to see that $\nu(k, m)$ attains integer values only for a finite number of hyperbolic pairs $(k, m)$. Since we will later deal with prime negative Euler characteristic while $|G|$ must be even, it will be sufficient to consider even integral values of $\nu(k, m)$ in (3.6). All the hyperbolic pairs $(k, m)$ with even $m$ for which $\nu=\nu(k, m)$ is an even integer are easily identified by hand and are listed in Table 1 with the proviso that pairs $(k, m)$ with both entries even are displayed only for $k \leq m$. 
We will use the information from Table 1 in the proof of the following result.

Proposition 4.1. Let $M=\operatorname{Map}(G ; a, z)$ be a bi-rotary map of a hyperbolic type $(k, m)$ for even $m$, with Euler characteristic $\chi=-p$ for an odd prime $p$. If $p$ is a divisor of $|G|$, then $p \in\{3,7\}$.

Proof. Let $p$ be a divisor of $|G|$; letting $\nu=\nu(k, m)$ we have $|G|=\nu p$ by (3.6). Suppose that $G$ has a unique (and hence necessarily normal) Sylow $p$ subgroup $K$. If $p$ divides neither $k$ nor $m$, then, letting $\bar{G}=G / K, \bar{a}=a K$ and $\bar{z}=z K$, the triple $(\bar{G} ; \bar{a}, \bar{z})$ defines a bi-rotary map $\bar{M}=\operatorname{Map}(\bar{G}, \bar{a}, \bar{z})$ of the same type, $(k, m)$, and the natural projection $G \mapsto \bar{G}$ induces a smooth $p$-to-1 cover $M \rightarrow \bar{M}$ of maps. By smoothness, for the Euler characteristic $\bar{\chi}=\chi(\bar{M})$ we have $\bar{\chi}=\chi / p=-1$. This, however, contradicts the findings of [3] by which there are no bi-rotary maps on a surface with Euler characteristic -1 . Observe that no prime $p \geq 7$ divides one of $k, m$ in Table 1 . For $p=5$ there are only two exceptions in Table 1 , for $k=5$, corresponding to $m=20, \nu=4,|G|=20$, or $m=4, \nu=20,|G|=100$. In both cases $G$ contains a unique (and hence normal) Sylow 5-subgroup $L$ (isomorphic to $C_{5}$ and $C_{5} \times C_{5}$, respectively) with $z$, the element of order 5 , contained in $L$. But then $|G / L|=4$ while $G / L=\langle a L\rangle$ is a group of order 2, a contradiction. We have thus proved:

Claim 1: If $p \geq 5$, then $G$ has more than one Sylow $p$-subgroup.

Let $G^{\prime}=[G, G]$ be the derived subgroup of $G$. Since our map is nonorientable, Lemma 3.1 shows that $G / G^{\prime}$ is a cyclic group generated by $\bar{z}=z G^{\prime}$ and hence of order a divisor of $k$. But, by Table 1 , for $k \neq 5$ no prime $p \geq 5$ divides $k$, and the case $(p, k)=(5,5)$ cannot occur, as we saw in the previous paragraph. It follows that $p$ divides $\left|G^{\prime}\right|$ and so $G^{\prime}$ contains a subgroup of order $p$. If this subgroup is unique, it would be characteristic in $G^{\prime}$ and hence normal in $G$, and as the Sylow $p$-subgroups of $G$ are cyclic of order $p$, this contradicts Claim 1. We thus proved another auxiliary result.

Claim 2: If $p \geq 5$, then $G^{\prime}$ has more than one Sylow $p$-subgroup.

Recall that by (3.6) we have $|G|=\nu p$, where $\nu=\nu(k, m)$ is an even integer. By Sylow theorems the number $n_{p}$ of Sylow $p$-subgroups of $G$ is a divisor of $\nu$ and $n_{p} \equiv 1 \bmod p$; in particular, $|G| \geq p^{2}$ if $n_{p}>1$. If $p=13$ or $17, G$ would contain a unique Sylow $p$-subgroup and if $p \geq 29$, we have $|G|=\nu p \leq 24 p<p^{2}$ and so $G$ would also contain a unique Sylow $p$-subgroup, contrary to Claim 1. To complete the proof it would be sufficient to exclude the primes $p \neq 7$ in the range $5 \leq p \leq 23$, which could be done by checking against known lists of small groups; we will, however, give more details.

From Table 1 combined with Claim 1 and the calculation above it follows that for every $p$ such that $5 \leq p \leq 23$ and $p \neq 7$ we must have $n_{p}=p+1$, $\nu=c_{p} n_{p}$ and $|G|=c_{p}(p+1) p$, where $c_{5} \in\{1,2,4\}, c_{11} \in\{1,2\}$, and $c_{19}=$ $c_{23}=1$. We now consider the interplay between $G$ and $G^{\prime}$.

It is well known that there are only two perfect groups (i.e. those satisfy$\operatorname{ing} G^{\prime}=G$ ) of order at most 120 , namely, $A_{5}$ and SL $(2,5)$, and by GAP's Small 
Groups Library [13], there are no perfect groups of size $p \nu=11 \cdot 12=132$, $11 \cdot 24=264,19 \cdot 20=380$ and $23 \cdot 24=552$. But $G$ cannot be isomorphic to $A_{5}$ since, by Table 1 for $\nu=12$, some elements of the corresponding orders $k$ or $m / 2$ are outside the set $\{2,3,5\}$ of non-trivial orders present in $A_{5}$. The other case when $G \cong \operatorname{SL}(2,5)$ would correspond to a map $M$ of type $(3,8)$. Letting $H \cong C_{2}$ be the centre of $G$, then $G / H \cong A_{5}$ and (considering the orders of elements in $A_{5}$ ) the induced quotient map $\bar{M}$ would have to be of type $(3,4)$. But then $\bar{M}$ would be an orientable map (on a sphere), a contradiction since a (branched) cover of a non-orientable map cannot be orientable.

In all the remaining cases to be considered we may assume that $\left|G^{\prime}\right|$ is a proper divisor of $|G|=c_{p}(p+1) p$. If $\left|G^{\prime}\right|$ is a proper divisor of $(p+1) p$, then the derived group $G^{\prime}$ would contain a unique Sylow $p$-subgroup, contrary to Claim 2. As $\left|G^{\prime}\right|$ now has to be a proper divisor of $(p+1) p$ for all $p$ in our range for which $c_{p}=1$, we only need to eliminate the cases when $c_{p} \in\{2,4\}$. Using the well-known fact that the only group of order 60 with six Sylow 5-subgroups is $A_{5}$ (which has already been excluded) the remaining cases to deal with for $c_{p} \in\{2,4\}$ are $p=11,|G|=2(p+1) p=24 \cdot 11$ and $p=5$, $|G|=4(p+1) p=24 \cdot 5$. In all these cases we have $k=3$, and all are eliminated by recalling that $\left|G / G^{\prime}\right|$ is a divisor of $k$ distinct from 1 and a divisor of $c_{p}$. This completes the proof.

With this in hand, we are in position to completely determine the birotary maps of Euler characteristic $-p$ for odd primes $p$ in the case when $p$ divides the order of the group, as the work for $p \in\{3,7\}$ has been done earlier in $[3]$.

Theorem 4.2. Let $M=\operatorname{Map}(G ; a, z)$ be a bi-rotary map of hyperbolic type $(k, m)$ for even $m$, with Euler characteristic $\chi=-p$ for an odd prime $p$. If $p$ is a divisor of $|G|$, then $p \in\{3,7\}$, and, up to isomorphism, $M$ is one of the three maps in Table 2 below, with partial presentations (3.4) completed by suitable additional relators.

To complete our classification project it thus remains to consider the case when the order of the group $G=\langle a, z\rangle$ with presentation (3.4) is not divisible by the odd prime $p$ equal to the negative of the Euler characteristic $\chi$ of the bi-rotary map $\operatorname{Map}(G ; a, z)$ of type $(k, m)$ and with $m$ even. Euler's formula (3.6) then implies (after cancellation of an obvious factor of 2) the existence of a positive integer $\lambda$ such that

TABLE 2. Bi-rotary maps with $p=-\chi$ an odd prime dividing $|G|$

\begin{tabular}{rrlll}
\hline$p$ & $|G|$ & $G$ & $(k, m)$ & Extra relators \\
\hline 3 & 18 & $C_{3} \times S_{3}$ & $(6,6)$ & {$[a, z] a z^{3}$} \\
3 & 36 & $\left(C_{3} \times C_{3}\right) \rtimes C_{4}$ & $(4,6)$ & $(a z)^{4},\left(a z^{2}\right)^{3}$ \\
7 & 168 & $\operatorname{PSL}(2,7)$ & $(3,8)$ & $(a z)^{7}$ \\
\hline
\end{tabular}




$$
\frac{k m}{2}-k-m=\lambda p \quad \text { and } \quad k m=\lambda|G| .
$$

From now on we let $y=[a, z]=a z a z^{-1}$ throughout; by Lemma 3.1 we have $G=\langle y, z\rangle$ as well. Note that $a y a=y^{-1}$ and recall that the orders of $z$ and $y$ are $k$ and $m / 2$. We begin with an auxiliary result for our automorphism groups of bi-rotary maps.

Lemma 4.3. Suppose that the group $G=\langle a, z\rangle=\langle y, z\rangle$ is, at the same time, a product $G=\langle a, y\rangle\langle z\rangle$ of two subgroups of coprime order, with odd $k>1$. Then, $\langle a, y\rangle$ is a normal subgroup of $G$ isomorphic to $C_{2} \times C_{2}$.

Proof. The result of Huppert [15] on solvability of a product of a dihedral and an Abelian group implies that such a group $G$ is solvable. In addition to this, $k$ and $m=|\langle a, y\rangle|$ are relatively prime. This readily implies that every oddorder Sylow subgroup of $G$ is cyclic and the Sylow 2-subgroup of $G$ is dihedral because of being contained in $\langle a, y\rangle$.

Solvable groups with this kind of structure of Sylow subgroups have been classified by Zassenhaus [23] and an alternative and somewhat more accessible form of this result was given by Wolf [22]. Instead of going through the lists given in [23] and [22] we adopt an approach using Fitting subgroups as developed in [10]. We recall that the Fitting subgroup $F$ of a group $H$ is the (unique) largest nilpotent normal subgroup of $H$, or, equivalently, the product of all nilpotent normal subgroups of $H$; it is obviously a characteristic subgroup of $H$. If, moreover, $H$ is solvable (which will be the case in our considerations), by e.g. $[18,5.4 .4]$ for the centralizer $C_{H}(F)$ and the centre $Z(F)$ one has $C_{H}(F)=Z(F) \leq F$. We saw that $G=\langle a, y\rangle\langle z\rangle$ is solvable and so its (characteristic) Fitting subgroup $F$ satisfies $C_{G}(F)=Z(F) \leq F$. The structure of Sylow subgroups of $G$ mentioned above together with the fact that $F$, being nilpotent, is a direct product of its Sylow subgroups implies that $F=F_{1} \times F_{2}$ where $F_{1}$ is a cyclic group of odd order and $F_{2}$ is a 2-group. We will consider two cases, depending on whether $F$ is cyclic or not.

Suppose that $F$ is cyclic, that is, $F_{2}$ is a cyclic group of order $2^{n}$ for some $n \geq 0$. Since $C_{G}(F)=Z(F) \leq F$, conjugation of $F$ by elements of $G$ induces a group homomorphism from $G$ into $\operatorname{Aut}(F)$ with kernel contained in $F$. The assumption that $F$ is cyclic implies that $F \leq C_{G}(F)$, which in combination with the previous inclusion implies that $F=C_{G}(F)=Z(F)$. This allows us to conclude that $G / F$ embeds into $\operatorname{Aut}(F)$. But in our case $\operatorname{Aut}(F)$ is Abelian and hence so is $G / F=\langle a F, z F\rangle=\langle y F, z F\rangle$. Further, commutativity of $G / F$ implies that $y F=1 F$, that is, $y \in F$, and so $\langle y\rangle=F_{2}$, of order $m / 2$. From $\langle a F, z F\rangle=\langle z F\rangle$ we obtain $a \in\langle z\rangle F=F\langle z\rangle$, which is a well-defined group as $F$ is normal in $G$. But since $z$ has odd order, all involutions of $F\langle z\rangle$ must live in $F$ and so $a \in F$ and in particular $a \in F_{2}$, that is, $a \in\langle y\rangle$, contrary to $\langle a, y\rangle$ being of order $m$.

We conclude that $F$ is not cyclic, that is, $F_{2}$ must be a dihedral 2-group (as customary, we consider $C_{2} \times C_{2}$ to be dihedral as well), and by Sylow theory we may assume that $F_{2}$ is a subgroup of $\langle a, y\rangle$. Since $F$ is normal in $G$, the 2-group $F_{2}$ is also normal in $G$ and hence normal in $\langle a, y\rangle$. But the latter 
is a dihedral group of order $m$ divisible by 4 , and its only normal dihedral subgroups are $\left\langle a, y^{2}\right\rangle,\left\langle a y, y^{2}\right\rangle$ if $m \geq 8$ and, of course, $\langle a, y\rangle$ for $m \geq 4$; these are the only three candidates for $F_{2}$. Let $K$ be any of the first two subgroups and suppose that $K$ is normal in $G$. Realizing that $G=\langle a, z\rangle=\langle y, z\rangle=\langle a y, z\rangle$ and forming $G / K$ we would obtain $K y \in K\langle z\rangle$ and so $y \in K\langle z\rangle$, contrary to $\langle a, y\rangle \cap\langle z\rangle=1$. We are thus left with the only possibility that $F_{2}=\langle a, y\rangle$, of order $m=2^{n}$ for some $n \geq 2$. Hence $\langle a, y\rangle$ is normal in $G$. If $n \geq 3$, then the subgroup $\langle y\rangle$ is characteristic in $F_{2}$ and hence normal in $G$, giving $G=\langle y, z\rangle=\langle y\rangle\langle z\rangle$, contradicting $|G|=m$. It follows that $n=2$ and so $m=4$ and $F_{2}=\langle a, y\rangle=C_{2} \times C_{2}$.

With Lemma 4.3 in hand we continue by looking at the intersection of the dihedral group $\langle a, y\rangle \cong D_{m}$ of order $m$ with the cyclic group $\langle z\rangle \cong C_{k}$.

Proposition 4.4. Let $\operatorname{Map}(G ; a, z)$ be a bi-rotary map of Euler characteristic $-p$ for an odd prime $p$, such that $p$ is not a divisor of $|G|$. Then, $\langle y\rangle \cap\langle z\rangle=1$, and for the integer $\lambda$ in (4.1) we only have the following two possibilities:

(a) $\lambda=2,\langle a, y\rangle \cap\langle z\rangle=\left\{1, z^{k / 2}\right\}$ with $a y^{t}=z^{k / 2}$ for some $t$, and $G=\langle y\rangle\langle z\rangle$, for $m \equiv 2 \bmod 4$, even $k \geq 4$, and $\operatorname{gcd}(k, m / 2)=1$, or

(b) $\lambda=1$, with $\langle a, y\rangle \cap\langle z\rangle=1$ and $G=\langle a, y\rangle\langle z\rangle$, for $m$ divisible by 4 , odd $k \geq 3$, and $\operatorname{gcd}(k, m)=1$.

Proof. Suppose that the (obviously cyclic) group $L=\langle y\rangle \cap\langle z\rangle$ contains a non-trivial element $w$. Since $G=\langle y, z\rangle, w$ must be central in $G$ and hence commuting with $a$, so that $w^{a}=w$. But as $w$ is a power of $y$ we also have $w^{a}=w^{-1}$, which implies that $w=w^{-1}$ and so $w$ has order 2 . As $L$ is an intersection of two cyclic groups, both $k$ and $m / 2$ must be even, $L \cong Z_{2}$ and the unique non-trivial element of $L$ has the form $w=y^{m / 4}=z^{k / 2}$. In this situation the left-hand side of the first equation of (4.1) is even and therefore $\lambda$ must be even as well.

We continue with the following useful observation: if $a=y^{i} z^{j}$ for some integers $i, j$, then $m / 2$ is odd. Indeed, rewriting $y=[a, z]=a z a z^{-1}$ in the form $y z a=a z$, substituting $a=y^{i} z^{j}$ and rearranging yields $z y^{i} z^{-1}=y^{i-1}$. This implies that two consecutive powers of $y$ have the same order, which is possible only if the order, $m / 2$, of $y$ is odd.

Combining the earlier conclusion that $4 \mid m$ with our observation, we conclude that the element $a \in G$ cannot be written in the form $a=y^{i} z^{j}$. In particular, $a \notin\langle y\rangle\langle z\rangle$ and the intersection $\langle a, y\rangle \cap\langle z\rangle$ contains no element of the form $y^{i} a$. The last fact and our findings about $L$ also imply that the intersection $L_{a}=\langle a, y\rangle \cap\langle z\rangle$ has order 2. From the second equation of (4.1) and elementary group theory we obtain

$$
\frac{k m}{\lambda}=|G| \geq \frac{|\langle a, y\rangle| \cdot|\langle z\rangle|}{\left|L_{a}\right|}=\frac{k m}{\left|L_{a}\right|}=\frac{k m}{2},
$$

which (recalling the parity of $\lambda$ ) implies that $\lambda=2$.

By the first part of (4.1) combined with divisibility of $m$ by 4 it follows that $k \equiv 2 \bmod 4$, and so $\langle y\rangle \cap\left\langle z^{2}\right\rangle=\langle a, y\rangle \cap\left\langle z^{2}\right\rangle=1$. A similar calculation as in (4.2) allows us to conclude that $\mathrm{km} / 2=|G|=|\langle a, y\rangle| \cdot\left|\left\langle z^{2}\right\rangle\right|$ and so 
$G=\langle a, y\rangle\left\langle z^{2}\right\rangle$. Consider the quotient group $Q=G / L=\langle a L, z L\rangle=\langle y L, z L\rangle$, of order $\frac{k m}{4}=2 \frac{k}{2} \frac{m}{4}$, the latter two factors being orders of $y L$ and $z L$ with odd $\frac{k}{2}>1$ (note that $k=2$ would imply $p=-1$ ). Since $\frac{m}{2}$ is even, we have $a \notin\langle y\rangle=\langle y\rangle L$ and so $a L \notin\langle y L\rangle$, which means that $|\langle a L, y L\rangle|=2 \frac{m}{4}=\frac{m}{2}$. Further, as $\operatorname{gcd}\left(\frac{k}{2}, \frac{m}{2}\right)=1$, it follows that $\langle a L, y L\rangle \cap\langle z L\rangle=1$ and therefore $|\langle a L, y L\rangle\langle z L\rangle|=\frac{m}{2} \frac{k}{2}=\frac{k m}{4}=|Q|$, that is, $Q=\langle a L, y L\rangle\langle z L\rangle$.

In this situation we may apply Lemma 4.3 and conclude that $\langle a L, y L\rangle$ is normal in $Q$, and as $L \subset\langle a, y\rangle$ this also means that $\langle a, y\rangle$ is also a normal subgroup of $G$. If the order $\frac{m}{2}$ of $y$ is equal to 2 , recalling the element $w$ from the beginning of the proof we would conclude that the group $G=\langle y, z\rangle=$ $\langle w, z\rangle=\langle z\rangle$ is Abelian, a contradiction. It follows that $\frac{m}{2}>2$ and so $\langle y\rangle$, being characteristic in the dihedral group $\langle a, y\rangle$, is a normal subgroup of $G$ as well. But then $G /\langle y\rangle=\langle z\langle y\rangle\rangle$ is cyclic, giving $a\langle y\rangle=z^{i}\langle y\rangle$, for some $i$, and hence $a \in\langle y\rangle\langle z\rangle$, a possibility that has already been excluded. This contradiction proves that $L$ is trivial.

Having established triviality of $L$ we may re-use some of the earlier arguments to prove more. Indeed, observe that $|L|=1$ implies that for the intersection $L_{a}=\langle a, y\rangle \cap\langle z\rangle$ we have $\left|L_{a}\right| \leq 2$ since $\langle z\rangle$ is cyclic. Now, using (4.2) without the right-most equality we obtain $\lambda \leq\left|L_{a}\right| \leq 2$.

If $\lambda=2$, then we immediately have $\left|L_{a}\right|=2$ by (4.2), and the only way to have this is that $a y^{t}=z^{k / 2}$ for some $t$. Note that $m / 2$ must then be odd by our observation, and $k$ must be even since $z^{k / 2} \in L_{a} ; \operatorname{also}, \operatorname{gcd}(k, m / 2)=1$ because the two integers have different parity and cannot have any common odd prime divisor by the first part of (4.1) for $\lambda=2$. Moreover, if $\lambda=2$ we clearly have $G=\langle y\rangle\langle z\rangle$.

If $\lambda=1$, the left-hand part of (4.1) written in the form $k(m / 2-1)-m=p$ implies that $4 \mid m$ and $k$ is odd, with $\operatorname{gcd}(k, m)=1$. By our earlier observation and arguments as above we then conclude that $L_{a}$ is trivial and $G=\langle a, y\rangle\langle z\rangle$. This establishes both (a) and (b); the inequalities for $k$ follow from the obvious fact that $(k, m)$ is a hyperbolic pair.

\section{The Classification}

Results of the previous section helped further reduce our classification project to consideration of the cases (a) and (b) of Proposition 4.4. We begin here with expanding on the observation used in the proof of this proposition in conjunction with the conclusion (a); note that $a y^{t}=z^{k / 2}$ is equivalent to $a=y^{t} z^{k / 2}$. In the second part of the statement below we have deliberately chosen to use a different notation for the group and its elements.

Proposition 5.1. Let $k$ and $m$ be integers such that $k$ is even, $k \geq 4, m \equiv 2$ $\bmod 4$, and $\operatorname{gcd}(k, m / 2)=1$.

(i) If $\operatorname{Map}(G ; a, z)$ is a bi-rotary map of type $(k, m)$ such that $G=\langle y\rangle\langle z\rangle$ with $\langle y\rangle \cap\langle z\rangle=1$ and $a=y^{t} z^{k / 2}$, then $G=\langle y\rangle \rtimes\langle z\rangle$ is a metacyclic group, $t$ and $t-1$ are units $\bmod m / 2$, and $z y z^{-1}=y^{r}$ for a unit $r$ 
such that $r-1$ is also a unit $\bmod m / 2$ and $t \equiv-(r-1)^{-1} \bmod m / 2$; moreover, $r^{k / 2} \equiv-1 \bmod m / 2$.

(ii) Conversely, if $H=\langle u\rangle \rtimes\langle v\rangle$ is a metacyclic group with $u$ and $v$ of order $m / 2$ and $k$ and with $v u v^{-1}=u^{r}$ for some unit $r \bmod m / 2$ such that $r-1$ is also a unit $\bmod m / 2$ and $r^{k / 2} \equiv-1 \bmod m / 2$, then $b=u^{t} v^{k / 2}$ for $t=-(r-1)^{-1}$ is an involution of $H$ and $\operatorname{Map}(H ; b, v)$ is a bi-rotary map of type $(k, m)$, with $u=b v b v^{-1}$.

Proof. For the part (i), by our observation in the proof of Proposition 4.4 we know that $a=y^{t} z^{k / 2}$ implies $z y^{t} z^{-1}=y^{t-1}$ and we concluded that equality of the orders of two consecutive powers of $y$ implied oddness of the order of $y$ (which is the case here as $m / 2$ is odd). In somewhat more detail, the orders of $y^{t}$ and $y^{t-1}$ are quotients of $m / 2$ divided by $\operatorname{gcd}(m / 2, t)$ and $\operatorname{gcd}(m / 2, t-1)$, respectively, and since the two gcd's are now equal to each other they must be equal to 1 by consecutiveness of $t$ and $t-1$. This allows us to make a stronger conclusion that the orders of $y, y^{t}$ and $y^{t-1}$ are equal to each other (and equal to $m / 2$ ); in particular, both $t$ and $t-1$ are units $\bmod m / 2$.

Since $y^{t}$ is a generator of $\langle y\rangle$, the relation $z y^{t} z^{-1}=y^{t-1}$ means that $\langle y\rangle$ is a normal subgroup of $G$. The orders $m / 2$ and $k$ of the groups $\langle y\rangle$ and $\langle z\rangle$ are now relatively prime and so, by the Schur-Zassenhaus theorem, the product $G=\langle y\rangle\langle z\rangle$ is a split extension of $\langle y\rangle$ by $\langle z\rangle$. In particular, there is a unit $r$ $\bmod m / 2$ such that $z y z^{-1}=y^{r}$.

Taking a $t$-th power of the last relation and comparing it with the earlier one gives $z y^{t} z^{-1}=y^{r t}=y^{t-1}$, which is equivalent to $t r \equiv t-1 \bmod m / 2$ and further to $(r-1) t \equiv-1 \bmod m / 2$. It follows that $r-1$ is also a unit $\bmod m / 2$ and $t=-(r-1)^{-1} \bmod m / 2$. Finally, $a^{2}=1$ is equivalent to $z^{k / 2} y^{t} z^{k / 2}=y^{-t}$ and since $y^{t}$ is a generator of $\langle y\rangle$ we also have $z^{k / 2} y z^{k / 2}=y^{-1}$. But from $z y z^{-1}=y^{r}$ we also have $z^{k / 2} y z^{k / 2}=y^{s}$ where $s=r^{k / 2}$. Comparing the last two expressions for the $z^{k / 2}$-conjugate of $y$ we conclude that $r^{k / 2} \equiv-1 \bmod$ $m / 2$.

We now prove the converse, that is, (ii). If $r$ is as in the statement of (ii), let $t \equiv-(r-1)^{-1} \bmod m / 2$; then $r t \equiv t-1 \bmod m / 2$. The assumption $r^{k / 2} \equiv$ $-1 \bmod m / 2$ implies that $v^{k / 2}$ conjugates every element of $\langle u\rangle$ to its inverse. In particular, $v^{k / 2} u^{t} v^{k / 2}=u^{-t}$, which implies that the element $b=u^{t} v^{k / 2}$ has order 2. Finally, since $v u v^{-1}=u^{r}$, it follows that $v u^{t} v^{-1}=u^{r t}=u^{t-1}$ and hence $v u^{-t} v^{-1}=u^{1-t}$. Now,

$$
\begin{aligned}
b v b v^{-1} & =u^{t} v^{k / 2} v u^{t} v^{k / 2} v^{-1}=u^{t} v\left(v^{k / 2} u^{t} v^{k / 2}\right) v^{-1}=u^{t} v u^{-t} v^{-1} \\
& =u^{t} u^{1-t}=u
\end{aligned}
$$

as claimed.

In the case (a) of Proposition 4.4 we are now ready to offer the corresponding classification result; note that the conditions of (a) mean that a vertex stabilizer and a face stabilizer of a bi-rotary map intersect non-trivially. To facilitate the statement, for each prime $p \equiv-1 \bmod 4$ we let $T_{p}$ denote the set of all ordered triples $(k, m, r)$ such that $(k, m)$ is a hyperbolic pair with $k$ 
even, $m \equiv 2 \bmod 4,(k-2)(m-2)=4(p+1)$, and both $r$ and $r-1$ are units $\bmod m / 2$ such that $r^{k / 2} \equiv-1 \bmod m / 2$.

Theorem 5.2. Let $M$ be a bi-rotary map of Euler characteristic $-p$ for an odd prime $p$ not dividing the number of arcs, and assume that a vertex stabilizer and a face stabilizer of $M$ intersect non-trivially. Then, $p \equiv-1 \bmod 4$, and $M$ is isomorphic, for some ordered triple $(k, m, r) \in T_{p}$, to a map $\operatorname{Map}(G ; a, z)$ with $G=\langle y\rangle \rtimes\langle z\rangle$ for $y=[a, z]$, presented in the form

$$
G=\left\langle a, z \mid a^{2}, z^{k}, y^{m / 2}, z y z^{-1} y^{-r}, a z^{k / 2} y^{t}\right\rangle
$$

where $t=(r-1)^{-1} \bmod m / 2$. Moreover, distinct triples from $T_{p}$ yield nonisomorphic bi-rotary maps.

Proof. Let $M=\operatorname{Map}(G ; a, z)$ be a bi-rotary map satisfying the above assumptions. Then $p$ is not a divisor of $|G|$, the number of $\operatorname{arcs}$ in $M$, and the non-trivial intersection of stabilizers implies that the conclusion (a) of Proposition 4.4 applies to $G, a, z$, and so does part (i) of Proposition 5.1. In particular, the value of $\lambda$ is equal to $2, k$ is even, and $m \equiv 2 \bmod 4$.

If $p \equiv 1 \bmod 4$, then, rewriting the first part of (4.1) in the form $(k-$ $2)(m-2)=4(p+1)$, one sees that $k$ would have to be divisible by 4 and $m \equiv 6 \bmod 8$, so that $m / 2 \equiv 3 \bmod 4$. By the item $r^{k / 2} \equiv-1 \bmod m / 2$ of part (i) of Proposition 5.1, however, we would conclude that -1 is a square modulo a number congruent to $3 \bmod 4$, which is impossible by elementary number theory. This proves that $p \equiv-1 \bmod 4$.

Using now the full power of part (i) of Proposition 5.1, by inspection one concludes that all the relators listed in the presentation (5.1) are indeed valid relators in $G$ for some triple $(k, m, r) \in T_{p}$. Part (ii) of Proposition 5.1 implies that (5.1) is indeed a presentation of a group of a bi-rotary map of type $(k, m)$ such that a vertex stabilizer and a face stabilizer intersect non-trivially, and hence in a subgroup of order 2 containing the involution $z^{k / 2}=a y^{-t}$ for $t=(r-1)^{-1} \bmod m / 2$.

Finally, if two maps $\operatorname{Map}\left(G_{i} ; a_{i}, z_{i}\right)$ as above for $i=1,2$ are isomorphic, then there would have to be a group isomorphism $G_{1} \rightarrow G_{2}$ taking $a_{1}$ to $a_{2}$ and $z_{1}$ to $z_{2}$, implying immediately that the maps would have the same type $(k, m)$ and the same exponent $r$ defining the semidirect product. This shows that bi-rotary maps as above that correspond to distinct triples from $T_{p}$ cannot be isomorphic.

Observe that the set $T_{p}$ is non-empty for every prime $p \equiv-1 \bmod 4$. Indeed, one easily checks that $(k, m, r)=(p+3,6,2) \in T_{p}$, and hence there is at least one bi-rotary map as referred to in Theorem 5.2 for every prime $p \equiv-1 \bmod 4$.

By part (b) of Proposition 4.4 the remaining case to be settled is when

$$
\begin{gathered}
G=\langle a, y\rangle\langle z\rangle,|G|=k m, k \geq 3 \text { odd }, 4 \mid m, \operatorname{gcd}(k, m)=1, \\
\text { with }\langle a, y\rangle \cap\langle z\rangle=1 .
\end{gathered}
$$


Proposition 5.3. Let $G$ be a group as in (5.2), where $G=\langle a, z\rangle=\langle y, z\rangle$. Then, $m=4, k \equiv 3 \bmod 6$, and $G$ is a semidirect product of the form $\langle a, y\rangle \rtimes\langle z\rangle \cong$ $\left(C_{2} \times C_{2}\right) \rtimes C_{k}$, with presentation

$$
G=\left\langle a, z \mid a^{2}, z^{k},[a, z]^{2}, z^{-1} a z[a, z]\right\rangle .
$$

Conversely, a group $G$ with such a presentation satisfies (5.2), with $y=[a, z]=$ $a z a z^{-1}$.

Proof. By Lemma 4.3 the group $\langle a, y\rangle$ is a normal subgroup of $G$ isomorphic to $C_{2} \times C_{2}$. The Schur-Zassenhaus theorem applied to the subgroups $\langle a, y\rangle$ and $\langle z\rangle$ of relatively prime orders (and the first one being normal in $G$ ) shows that $G$ is a semidirect product

$$
\langle a, y\rangle \rtimes\langle z\rangle \cong\left(C_{2} \times C_{2}\right) \rtimes Z_{k} .
$$

It remains to specify the homomorphism $\langle z\rangle \rightarrow \operatorname{Aut}\left(C_{2} \times C_{2}\right) \cong S_{3}$ given by conjugation of $\langle a, y\rangle$ by $z$. Note that $z$ cannot commute with $y$ since otherwise $\langle y, z\rangle=\langle y\rangle\langle z\rangle$ and $G$ would have order $2 k$, a contradiction. Since $y=[a, z]=$ $a z a z^{-1}$ implies that $z a z^{-1}=a y$, the only possibility is that conjugation by $z$ induces a 3 -cycle on the non-identity elements of $\langle a, y\rangle$, that is, $z(a y) z^{-1}=y$, and $z y z^{-1}=a$. The last two relations are equivalent, since $y^{2}=1$ implies that

$$
z(a y) z^{-1}=y=y^{-1}=[a, z]^{-1}=z a z^{-1} a,
$$

from which one obtains $z y z^{-1}=a$, and the argument is reversible. This gives the presentation in the statement of our proposition.

The fact that this presentation indeed defines a group $G$ of order $4 k$ follows from the above analysis. (For completeness, note that the relation $[a, y]=1$ can be obtained from $z(a y) z^{-1}=y$ and its inverse.) Since the image of the homomorphism given by conjugation by $z$ is a 3 -cycle, $k$ must be divisible by 3 , and taking into account oddness of $k$ we obtain $k \equiv 3$ $\bmod 6$.

Using again the language of vertex stabilizers and face stabilizers, we are in position to state and prove the remaining part of our classification.

Theorem 5.4. Let $M$ be a bi-rotary map of Euler characteristic $-p$ for an odd prime $p$ not dividing the number of arcs, and assume that a vertex stabilizer and a face stabilizer of $M$ intersect trivially. Then, $p \equiv-1 \bmod 6$, and $M$ is isomorphic to a unique map $\operatorname{Map}(G ; a, z)$ of type $(p+4,4)$, with $G \cong\left(C_{2} \times\right.$ $\left.C_{2}\right) \rtimes C_{p+4}$ presented in the form

$$
G=\left\langle a, z \mid a^{2}, z^{p+4},[a, z]^{2}, z^{-1} a z[a, z]\right\rangle .
$$

Proof. The trivial intersection of stabilizers now implies that the conclusion (b) of Proposition 4.4 applies to $G, a, z$, and so $G=\langle a, z\rangle$ is a group as in (5.2). By Proposition 5.3 $G$ has a presentation as in (5.3) for $k \equiv 3 \bmod 6$ and $m=4$. The first part of $(4.1)$ gives $(k-2)(m-2)=2 p+4$ and as $m=4$ we obtain $k=p+4$, which, by $k \equiv 3 \bmod 6$, means that $p \equiv-1 \bmod 6$. 


\section{Summary and Remarks}

Let $M=\operatorname{Map}(G ; a, z)$ be a (finite) bi-rotary map of type $(k, m)$ for even $m$, with $G$ presented in the form $\left\langle a, z \mid a^{2}, z^{k},[a, z]^{m / 2}, \ldots\right\rangle$ as usual. In Sect. 3 we saw that $M$ arises as a (finite) smooth epimorphic image of the index-two subgroup $\Delta^{b}(k, m)$ of the full triangle group

$$
\Delta(k, m)=\left\langle R_{0}, R_{1}, R_{2} \mid R_{0}^{2}, R_{1}^{2}, R_{2}^{2},\left(R_{0} R_{2}\right)^{2},\left(R_{2} R_{1}\right)^{k},\left(R_{1} R_{0}\right)^{m}\right\rangle,
$$

generated by the elements $A=R_{0}$ and $Z=R_{1} R_{2}$, with full presentation

$$
\Delta^{b}(k, m)=\left\langle A, Z \mid A^{2}, Z^{k},[A, Z]^{m / 2}\right\rangle,
$$

under the natural epimorphism induced by the assignment $A \mapsto a$ and $Z \mapsto z$. Observe that $\Delta(k, m)$ can be looked at as an extension of $\Delta^{b}(k, m)$ by the involution $R_{2}$, in such a way that conjugation by $R_{2}$ preserves $A$ and inverts $Z$.

Recalling from Sect. 3 the tessellation $U(k, m)$ of a simply connected surface by $m$-gons, $k$ of which meet at every vertex, the element $R_{2}$ preserves $U(k, m)$ but inverts the bi-rotary local orientations around vertices. If the involution $R_{2}$ projects onto an involution $c$ extending the group $G=\langle a, z\rangle$ in such a way that conjugation by $c$ preserves $a$ and inverts $z$, then $c$ would induce a vertex, edge and face preserving mapping on $M$ that inverts the bi-rotary local orientations at vertices of $M$. We will call such a mapping a reflection, and a bi-rotary map admitting a reflection will be called reflexible. Thus, a bi-rotary map is reflexible if and only if it is fully regular (in the terminology introduced in Sect. 3); a bi-rotary map that is not fully regular will be called chiral, in analogy with chiral orientably regular maps.

An obvious equivalent way of checking if a bi-rotary map $M=\operatorname{Map}(G ; a, z)$ is reflexible is to perform the following test. Given a presentation of $G=\langle a, z\rangle$ in terms of relators over the alphabet $\{a, z\}$, for every word $W$ over $\{a, z\}$, let $W^{\prime}$ be the word obtained from $W$ by keeping the element $a$ unchanged and replacing every occurrence of $z$ by $z^{-1}$. Then, the map $M$ is reflexible if and only if, for every relator $R$ of the presentation of $G$, the word $R^{\prime}$ is also a relator of $G$.

Performing this test on the bi-rotary maps described in the previous sections readily shows that all the bi-rotary maps from Theorem 5.4 and the three ones from Theorem 4.2 are reflexible. The situation is slightly more complicated for the bi-rotary maps $\operatorname{Map}(G ; a, z)$ from Theorem 5.2 by means of presentations in (5.1). Let us focus on the relator $R=z y z^{-1} y^{-r}$ of (5.1) with $y=[a, z]=a z a z^{-1}$. Conjugating $R$ by $a z^{-1}$ and letting $y^{\prime}=a z^{-1} a z=$ $a z^{-1} y z a$ gives, after cancellation, the relator $z a z^{-1} a\left(y^{\prime}\right)^{-r}$, which is equal to $z y^{\prime} z^{-1}\left(y^{\prime}\right)^{-r}$. A comparison with $R$ implies that the last relator coincides with $R^{\prime}$ if and only if $z y^{\prime} z^{-1}=z^{-1} y^{\prime} z$, which happens to be equivalent to $\left[y, z^{2}\right]=1$. Conjugation of $z y z^{-1}=y^{r}$ by $z$ then gives $r^{2} \equiv 1 \bmod m / 2$. Since the condition $r^{k / 2} \equiv-1 \bmod m$ is part of the description of the maps in Theorem 5.2, we conclude that $k / 2$ must be odd and $r=-1$. Under these conditions the remaining relators of the presentation (5.1) are easily seen to transform to relators of $G$ under the transformation $a \mapsto a$ and $z \mapsto z^{-1}$. This shows that a bi-rotary map from Theorem 5.2 is reflexible if and only if $r=-1$ 
TABLE 3. Classification of bi-rotary maps with $\chi=-p$

\begin{tabular}{llllll}
\hline$-\chi$ & Type & $|G|$ & $G$ & $\begin{array}{l}\text { Additional } \\
\text { relators }\end{array}$ & Conditions \\
\hline 3 & $(6,6)$ & 18 & $C_{3} \times S_{3}$ & {$[a, z] a z^{3}$} & - \\
3 & $(4,6)$ & 36 & $\left(C_{3} \times C_{3}\right) \rtimes C_{4}$ & $(a z)^{4},\left(a z^{2}\right)^{3}$ & - \\
7 & $(3,8)$ & 168 & $\mathrm{PSL}(2,7)$ & $(a z)^{7}$ & - \\
$p$ & $(p+4,4)$ & $4(p+4)$ & $\left(C_{2} \times C_{2}\right) \rtimes C_{p+4}$ & $z^{-1} a z[a, z]$ & $p \equiv-1 \bmod 6$ \\
$p$ & $(k, m)$ & $k m / 2$ & $C_{m / 2} \rtimes C_{k}$ & $z y z^{-1} y^{-r}$, & $p \equiv 3 \bmod 4$, \\
& & & $a z^{k / 2} y^{s_{r}}$ & $(k, m, r) \in T_{p}$ \\
\hline
\end{tabular}

and $k \equiv 2 \bmod 4$. Note that this gives for every $p \equiv 3 \bmod 4$ at least one such reflexible bi-rotary map. Observe also that the first map of type $(6,6)$ from Theorem 4.2 also appears among the maps covered by Theorem 5.4.

In the light of the above discussion we may now give a summary of our classification results, parts of which have been given in Theorems 4.2, 5.2 and 5.4 .

Theorem 6.1. Let $M$ be a bi-rotary map of Euler characteristic $\chi=-p$ for an odd prime $p$, with automorphism group $G=$ Aut $^{b}(M)$ preserving local orientations. Then, $M$ is isomorphic to a map $\operatorname{Map}(G ; a, z)$ of type $(k, m), m$ even, for one of the group presentations $G=\left\langle a, z \mid a^{2}, z^{k},[a, z]^{m / 2}, \ldots\right\rangle$ in Table 3 below, with $y=[a, z]=a z a z^{-1}$ and with extra relators as listed. Further, in Table $3, T_{p}$ for $p \equiv 3 \bmod 4$ is the set of all ordered triples $(k, m, r)$ such that $(k, m)$ is a hyperbolic pair for even $k, m \equiv 2 \bmod 4,(k-2)(m-2)=4(p+1)$, with $r$ and $r-1$ being units $\bmod m / 2$ such that $r^{k / 2} \equiv-1 \bmod m / 2$ and $s_{r}=(r-1)^{-1} \bmod m / 2$ :

Moreover, all the maps appearing in the first four rows of the table are reflexible, and a map appearing in the fifth row is reflexible if and only if $k \equiv 2$ $\bmod 4$ and $r=-1$.

As mentioned in the Introduction, fully regular maps of negative prime Euler characteristic have been classified in [4]; see also [11] for a different proof of the same result. It is therefore of interest to compare our classification results of Theorem 6.1 for regular maps obtained as reflexible bi-rotary maps with those of [4] for regular maps in general. From Sect. 3 we know that the latter, of the form $\operatorname{Map}\left(G ; r_{0}, r_{1}, r_{2}\right)$, are given by presentations of the type (3.2), that is,

$$
G=\left\langle r_{0}, r_{1}, r_{2} \mid r_{0}^{2}, r_{1}^{2}, r_{2}^{2},\left(r_{0} r_{2}\right)^{2},\left(r_{1} r_{2}\right)^{k},\left(r_{0} r_{1}\right)^{m}, \ldots\right\rangle
$$

To present the results of [4] in a tabular form we will use the notation $r=r_{0} r_{1}$ and $s=r_{1} r_{2}$ of [20]. For primes $p \equiv 3 \bmod 4$, we also let $T_{p}^{\prime}$ be the set of all ordered pairs $(k, m)$ of integers congruent to $2 \bmod 4$ such that $(k-2)(m-2)=4(p+1)$. In these terms the classification of [4] of pairwise non-isomorphic fully regular maps $\operatorname{Map}\left(G ; r_{0}, r_{1}, r_{2}\right)$ of Euler characteristic $\chi=-p$ for odd primes $p$ and various types $(k, m)$ in terms of presentations of 
TABLE 4. Classification of fully regular maps with $\chi=-p$ from $[4]$

\begin{tabular}{llllll}
\hline$-\chi$ & Type & $|G|$ & $G$ & Additional relators & Conditions \\
\hline 3 & $(4,6)$ & 72 & $\left(C_{3} \times C_{3}\right) \rtimes D_{4}$ & $\left(r^{2} s^{2}\right)^{2}, s\left(r s^{-1}\right)^{3} r_{1}$ & + dual map \\
3 & $(5,4)$ & 120 & $S_{5}$ & $\left(s^{-1} r\right)^{3} r r_{1}$ & + dual map \\
3 & $(5,5)$ & 60 & $A_{5}$ & $\left(r s^{-1}\right)^{3}, s r^{2} s^{2} r r_{1}$ & - \\
5 & $(6,4)$ & 120 & $S_{5}$ & $\left(r s^{-1}\right)^{3},\left(s^{2} r^{2}\right)^{2} s r^{-1} r_{1}+$ dual map \\
7 & $(3,8)$ & 336 & $\operatorname{PGL}(2,7)$ & $\left(r^{-2} s\right)^{3} r^{-1} s r_{1}$ & + dual map \\
7 & $(3,8)$ & 336 & $\operatorname{PGL}(2,7)$ & $\left(r^{2} s^{-1}\right)^{4},\left(r^{3} s^{-1}\right)^{3} r r_{1}$ & + dual map \\
13 & $(3,7)$ & 1092 & $\operatorname{PSL}(2,13)$ & $\left(\left(s r^{-2}\right)^{4} s r^{3}\right)^{2}$ & + dual map \\
$p$ & $(p+4,4)$ & $8(p+4)$ & $C_{p+4} \cdot D_{8}$ & $r s^{3} r^{-1} s^{3}$ & + dual; $p \equiv 5$ \\
& & & & & $\begin{array}{l}\text { mod } 6 \\
\left(k s^{-1}\right)^{2},\end{array}$ \\
$p$ & $(k, m)$ & $k m$ & $D_{k} \times D_{m}$ & $r^{m / 2} s^{k / 2} r_{1}$ & $(k, m) \in T_{p}^{\prime}$ \\
\hline
\end{tabular}

their automorphism groups as above is given in the following table. (Note that the last two lines still include a map for $p=3$ and a map for $p=5$; additional relators in the first 6 lines are from [7], and the fourth line also includes an omission in [4] for $p=5$.)

To compare the results in Table 4 with those from Table 3 of Theorem 6.1 for reflexible bi-rotary maps, observe that our 'canonical' generators $a=r_{0}$, $z=r_{1} r_{2}$ and $y=[a, z]$ together with the 'reflection' involution $c=r_{2}$ of a reflexible bi-rotary map relate to the generators $r$ and $s$ from Table 4 by $r=a z c, s=z$, and $r^{2}=y=[a, z]$. Also, when comparing presentations one has to bear in mind that one needs to take extensions

$$
G\langle c\rangle=\left\langle a, c, z \mid a^{2}, c^{2}, z^{k},[a, c],(c z)^{2}, \ldots\right\rangle
$$

of the groups in Table 3 by adjoining the reflection $c$ and check these against Table 4.

With this in hand one may verify that the reflexible bi-rotary maps for $p=3$ and 7 from the second and the third row of Table 3 are isomorphic to the maps in the first and the fifth row of Table 4, with the additional relators $(a z)^{4},\left(a z^{2}\right)^{3}$ corresponding to $\left(r^{2} s^{2}\right)^{2}, s\left(r s^{-1}\right)^{3} r_{1}$ for $p=3$, and $(a z)^{7}$ corresponding to $\left(r^{-2} s\right)^{3} r^{-1} s r_{1}$ for $p=7$. Similarly, the reflexible birotary maps for $p \equiv-1 \bmod 6$ from the fourth row of Table 3 are isomorphic to the maps in the penultimate row of Table 4. Finally, the reflexible bi-rotary maps for $p \equiv-1 \bmod 4$ from the last line of Table 3 , that is, those for which $k \equiv 2 \bmod 4$ and $r=-1$, are isomorphic to those described in the last row of Table 4 , with the relators $z y z^{-1} y$ and $a z^{k / 2} y^{s_{r}}$ corresponding to $\left(r s^{-1}\right)^{2}$ and $r^{m / 2} s^{k / 2} r_{1}$ after the substitution as indicated.

These findings immediately imply the following unexpected consequence.

Corollary 6.2. For every prime $p>13$ and every fully regular map on a surface with Euler characteristic - p, either the map or its dual is a reflexible bi-rotary map. 
In view of this result, classification of bi-rotary maps on further families of surfaces, such as those of Euler characteristic $-p^{2},-2 p$ and $-3 p$, may lead to interesting conclusions when compared with the corresponding classification results of $[1,9-11]$ for regular maps.

\section{Acknowledgements}

Research of the first two authors was supported in part by the Portuguese Foundation for Science and Technology (FCT-Fundação para a Ciência e a Tecnologia), through CIDMA - Center for Research and Development in Mathematics and Applications, within project UID/MAT/04106/2019. Research of the third author was supported by the research grants APVV 17-0428 and APVV-15-0220, as well as the research grants VEGA 1/0026/16 and VEGA $1 / 0142 / 17$.

Open Access. This article is distributed under the terms of the Creative Commons Attribution 4.0 International License (http://creativecommons.org/licenses/ by/4.0/), which permits unrestricted use, distribution, and reproduction in any medium, provided you give appropriate credit to the original author(s) and the source, provide a link to the Creative Commons license, and indicate if changes were made.

Publisher's Note Springer Nature remains neutral with regard to jurisdictional claims in published maps and institutional affiliations.

\section{References}

[1] Belolipetsky, M., Jones, G.A.: Automorphism groups of Riemann surfaces of genus $p+1$, where $p$ is prime. Glasg. Math. J. 47(2), 379-393 (2005)

[2] Breda d'Azevedo, A.: A theory of restricted regularity of hypermaps. J. Korean Math. Soc. 43(5), 991-1018 (2006)

[3] Breda d'Azevedo, A., Catalano, D.A., Duarte, R.: Regular pseudo-oriented maps and hypermaps of low genus. Discrete Math. 338(6), 895-921 (2015)

[4] Breda d'Azevedo, A., Nedela, R., Širáň, J.: Classification of regular maps of negative prime Euler characteristic. Trans. Amer. Math. Soc. 357(10), 41754190 (2005)

[5] Bryant, R.P., Singerman, D.: Foundations of the theory of maps on surfaces with boundary. Quart. J. Math. Oxford Ser. (2) 36(141), 17-41 (1985)

[6] Conder, M.D.E.: Regular maps of Euler characteristic -1 to -600 . See webpages linked from http://www.math.auckland.ac.nz/ conder

[7] Conder, M.D.E., Dobcsányi, P.: Determination of all regular maps of small genus. J. Combin. Theory Ser. B 81(2), 224-242 (2001) 
[8] Conder, M.D.E., Hucíková, V., Nedela, R., Širáň, J.: Chiral maps of given hyperbolic type. Bull. Lond. Math. Soc. 48(1), 38-52 (2016)

[9] Conder, M.D.E., Nedela, R., Širán̆, J.: Classification of regular maps of Euler characteristic $-3 p$. J. Combin. Theory Ser. B. 102(4), 967-981 (2012)

[10] Conder, M.D.E., Potočnik, P., Širáň, J.: Regular maps with almost Sylow-cyclic automorphism groups, and classification of regular maps with Euler characteristic $-p^{2}$. J. Algebra 324(10), 2620-2635 (2010)

[11] Conder, M.D.E., Širáň, J., Tucker, T.W.: The genera, reflexibility and simplicity of regular maps. J. Eur. Math. Soc. 12(2), 343-364 (2010)

[12] Coxeter, H.S.M., Moser, W.O.J.: Generators and Relations for Discrete Groups. Fourth Edition. Springer-Verlag, Berlin-New York (1980)

[13] The GAP group: GAP - Groups, Algorithms, and Programming. Available at http://www.gap-system.org/

[14] Gorenstein, D., Walter, J.H.: The characterization of finite groups with dihedral Sylow 2-subgroups. Part I, J. Algebra 2, 85-151 (1965); Part II, J. Algebra 2, 218-270 (1965)

[15] Huppert, B.: Über die Aufösbarkeit faktorisierbarer Gruppen. Math. Z. 59, 1-7 (1953)

[16] Jones, G.A., Singerman, D.: Theory of maps on orientable surfaces. Proc. London Math. Soc. (3) 37(2), 273-307 (1978)

[17] Jones, G.A., Singerman, D.: Belyŭ functions, hypermaps and Galois groups. Bull. London Math. Soc. 28(6), 561-590 (1996)

[18] Robinson, D.J.S.: A Course in the Theory of Groups. Second Edition. Graduate Texts in Mathematics, 80. Springer-Verlag, New York (1996)

[19] Rose, H.E.: A Course on Finite Groups. Springer-Verlag London, Ltd., London (2009)

[20] Širán̆, J.: How symmetric can maps on surfaces be? In: Blackburn, S.R., Gerke, S., Wildon, M. (eds.) Surveys in Combinatorics 2013, pp. 161-238. London Math. Soc. Lecture Note Ser., 409. Cambridge Univ. Press, Cambridge (2013)

[21] Wilson, S.E.: Riemann surfaces over regular maps. Canad. J. Math. 30(4), 763782 (1978)

[22] Wolf, J.A.: Spaces of Constant Curvature. McGraw-Hill Book Co., New YorkLondon-Sydney (1967)

[23] Zassenhaus, H.: Über endliche Fastkörper. Abh. Math. Sem. Univ. Hamburg 11(1), 187-220 (1935) 
Antonio Breda d'Azevedo and Domenico A. Catalano Departamento de Matemática

Universidade de Aveiro

Aveiro

Portugal

e-mail: breda@ua.pt

Domenico A. Catalano

e-mail: domenico@ua.pt

Jozef Širán̆

Department of Mathematics and Statistics

The Open University

Milton Keynes MK7 6AA

UK

and

Department of Mathematics and Descriptive geometry Slovak University of Technology in Bratislava

Bratislava

Slovakia

e-mail: jozef.siran@open.ac.uk; jozef.siran@stuba.sk

Received: 7 October 2017.

Accepted: 11 February 2018. 